\begin{tabular}{|l|c|c|c|c|}
\hline \hline Patients with chills/rigors & $4(8 \%)$ & $22(47 \%)$ & $59(20 \%)$ & $165(56 \%)$ \\
\hline Patients with nausea & $4(8 \%)$ & $4(9 \%)$ & $38(13 \%)$ & $31(10 \%)$ \\
\hline Patients with vomiting & $2(4 \%)$ & $7(15 \%)$ & $19(6 \%)$ & $21(7 \%)$ \\
\hline Patients with other reactions & $10(21 \%)$ & $13(28 \%)$ & $47(16 \%)$ & $69(23 \%)$ \\
\hline
\end{tabular}

tDay 1 body temperature increased above the temperature taken within 1 hour prior to infusion (preinfusion temperature) or above the lowest infusion value (no preinfusion temperature recorded).

Less Common Adverse Events

The following adverse events also have been reported in $2 \%$ to $10 \%$ of AmBisome-treated patients receiving chemotherapy or bone marrow transplantation or had HIV disease in six comparative, clinical trials:

- Body as a Whole: abdomen enlarged, allergic reaction, cellulitis, cell mediated immunological reaction, face edema, graft versus host disease, malaise, neck pain, and procedural complication

- Cardiovascular System: arrhythmia, atrial fibrillation, bradycardia, cardiac arrest, cardiomegaly, hemorrhage, postural hypotension, valvular heart disease, vascular disorder, and vasodilatation (flushing) - Digestive System: anorexia, constipation, dry mouth/nose, dyspepsia, dysphagia, eructation, fecal incontinence, flatulence, hemorrhoids, gum/oral hemorrhage, hematemesis, hepatocellular damage, hepatomegaly, ileus, liver function test abnormal, mucositis, rectal disorder, stomatitis, ulcerative stomatitis, and veno-occlusive liver disease

- Hemic and Lymphatic System: anemia, coagulation disorder, ecchymosis, fluid overload, petechia, prothrombin decreased, prothrombin increased, and thrombocytopenia

- Metabolic and Nutritional Disorders: acidosis, amylase increased, hyperchloremia, hyperkalemia, hypermagnesemia, hyperphosphatemia, hyponatremia, hypophosphatemia, hypoproteinemia, lactate dehydrogenase increased, nonprotein nitrogen (NPN) increased, and respiratory alkalosis

- Musculoskeletal System: arthralgia, bone pain, dystonia, myalgia, and rigors

- Nervous System: agitation, coma, convulsion, depression, dysesthesia, dizziness, hallucinations, nervousness, paresthesia, somnolence, thinking abnormality, and tremor

- Respiratory System: asthma, atelectasis, hemoptysis, hiccup, hyperventilation, influenza-like symptoms, lung edema, pharyngitis, pneumonia, respiratory insufficiency, respiratory failure, and sinusitis

- Skin and Appendages: alopecia, dry skin, Herpes simplex, injection site reactions involving pain and inflammation, maculopapular rash, purpura, skin discolouration, skin disorder, skin ulcer, urticaria, and vesiculobullous rash

- Special Senses: conjunctivitis, dry eyes, and eye hemorrhage

- Urogenital System: abnormal renal function, acute kidney failure, acute renal failure, dysuria, kidney failure, toxic nephropathy, urinary incontinence, and vaginal hemorrhage

\section{Post-Market Adverse Drug Reactions}

The following adverse experiences have been reported infrequently in post-marketing surveillance, in addition to those mentioned above: agranulocytosis, anaphylactic reaction, angioedema, bronchospasm/wheezing, cholestasis, cyanosis/hypoventilation, erythema, epilepsy, fever, generalized edema, headache, hemorrhagic cystitis, hyperbilirubinemia, hypocalcemia, jaundice, increased liver enzymes, leukopenia, multi-organ failure, myocardial infarction, pelvic bleeding, pulmonary edema, renal impairment, retrosternal pain, stomach pain, sweating, swelling (face, lips, eyes), tachycardia, urea increase, urticaria.

\section{DOSAGE AND ADMINISTRATION}

\section{Special Patient Groups}

Renal Impairment: The effect of renal impairment on the disposition of AmBisome has not been studied. AmBisome has been successfully administered to patients with pre-existing renal impairment. For renal dialysis patients, AmBisome administration should be initiated after dialysis is completed.

Hepatic Impairment: The effect of hepatic impairment on the disposition of AmBisome is unknown. OVERDOSAGE

The toxicity of AmBisome due to overdose has not been defined. A maximum tolerated dose was not observed with repeated daily doses up to $10 \mathrm{mg} / \mathrm{kg}$ in pediatric patients and $15 \mathrm{mg} / \mathrm{kg}$ in adult patients. If an overdose is suspected, discontinue therapy, monitor the patient's clinical status and administer supportive therapy as required. Particular attention should be given to monitoring renal function.

\section{STORAGE AND STABILITY}

Storage of AmBisome

Unopened vials of lyophilized material are to be stored between temperatures of 2 to $25^{\circ} \mathrm{C}\left(36-77^{\circ} \mathrm{F}\right)$.

\section{Storage of Reconstituted Product Concentrate}

The reconstituted product concentrate may be stored for up to 24 hours at $2-8^{\circ} \mathrm{C}\left(36-46^{\circ} \mathrm{F}\right)$ following reconstitution with Sterile Water for Injection USP. Do not freeze.

Storage of Reconstituted Product Diluted with 5\% Dextrose

Do not freeze. Injection of AmBisome should commence within 6 hours of dilution with $5 \%$ Dextrose.

CAUTION: DISCARD partially used vials.

SPECIAL HANDLING INSTRUCTIONS

Not Applicable.

\section{DOSAGE FORMS, COMPOSITION AND PACKAGING}

AmBisome is supplied as a lyophilized powder in either $20 \mathrm{ml}$ or $30 \mathrm{ml}$ vials. Each vial of AmBisome contain $50 \mathrm{mg}$ of amphotericin B. AmBisome for Injection is available as single unit vials in a pack of ten vials in individual cartons. Each carton contains one pre-packaged, disposable sterile 5 micron filter.

Full Product Monograph available on request.

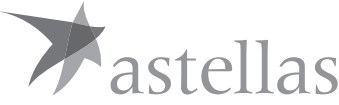

Markham, Ontario L3R 0B8

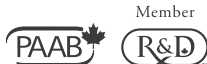

AmBisome $^{\circledast}$ is a registered trademark of Astellas Pharma, Inc., Tokyo, Japan.

\section{ADVERTISERS' INDEX}

The Canadian Journal of Infectious Diseases $\mathbb{E}$ Medical Microbiology

is a peer-reviewed journal, financed almost entirely through advertising.

The companies advertising in the Journal

recognize the need to support high-quality,

Canadian, ethical journals and to promote the publishing of Canadian medical research.

The support of the following companies is appreciated:

\section{Abbott Laboratories}

Biaxin XL................IBC

Astellas Pharma

AmBisome ................. IFC

Prescribing information. ...........446

BD Medical

Corporate...................393

Pfizer Canada

Eraxis/Vfend/Zyvoxam........... OBC

\section{ERRATUM}

Please note that errors was made in the January/ February 2008 issue of The Canadian Journal of Infectious Diseases $\mathcal{E}$ Medical Microbiology, in the AMMI Canada Guidelines titled "Clinical practice guidelines for hospital-acquired pneumonia and ventilator-associated pneumonia in adults" (Can J Infect Dis Med Microbiol 2008;19[1]:19-53).

The authors apologize for these errors.

On pages 29 and 30, all instances in which the text states that "the CPIS is less than 6" or "below 6" should be revised to "6 or less" to be consistent with Figure 5. 


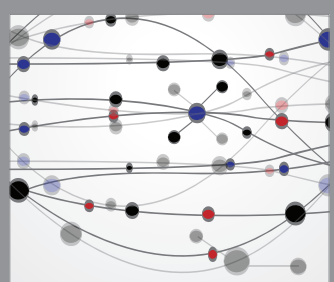

The Scientific World Journal
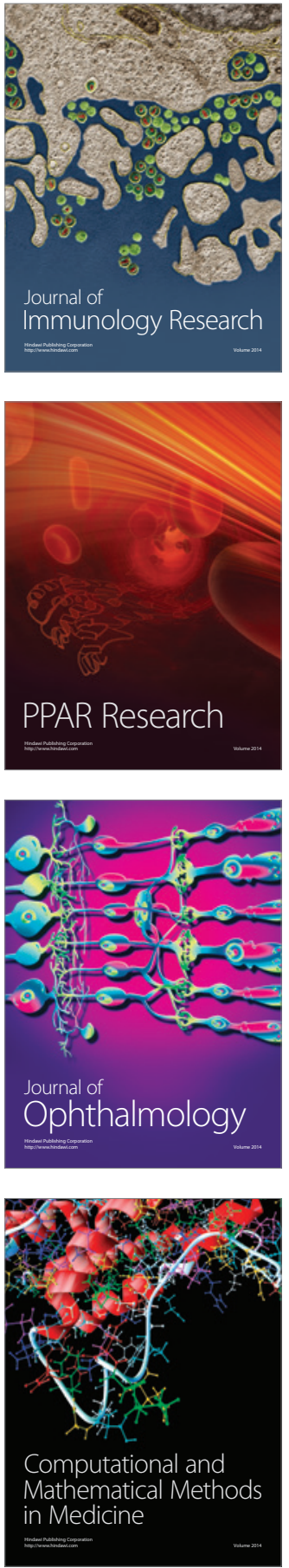

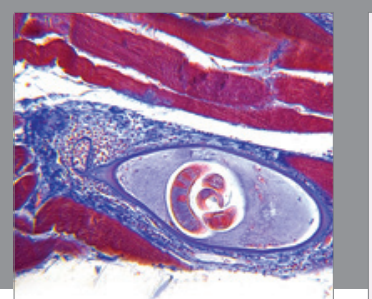

Gastroenterology Research and Practice

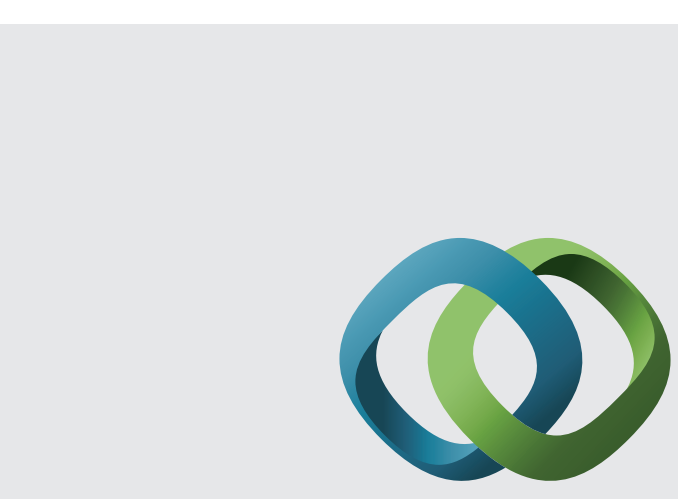

\section{Hindawi}

Submit your manuscripts at

http://www.hindawi.com
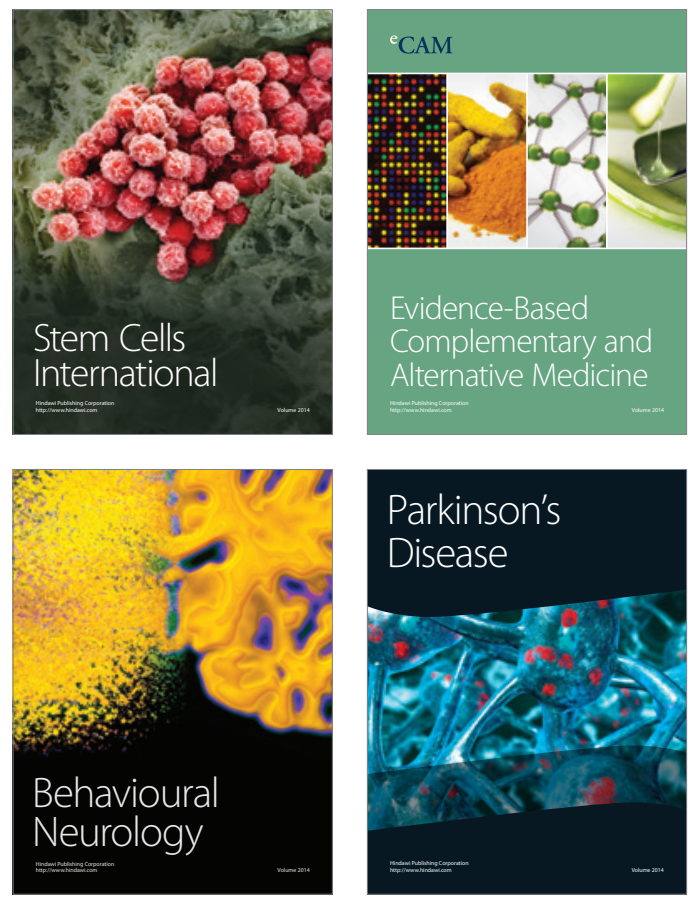
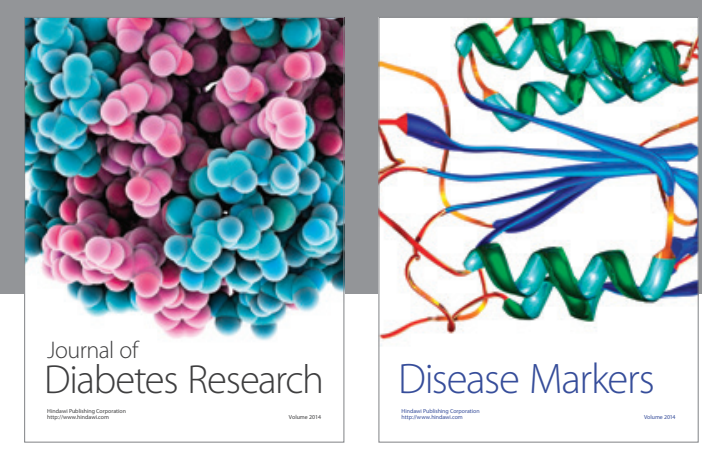

Disease Markers
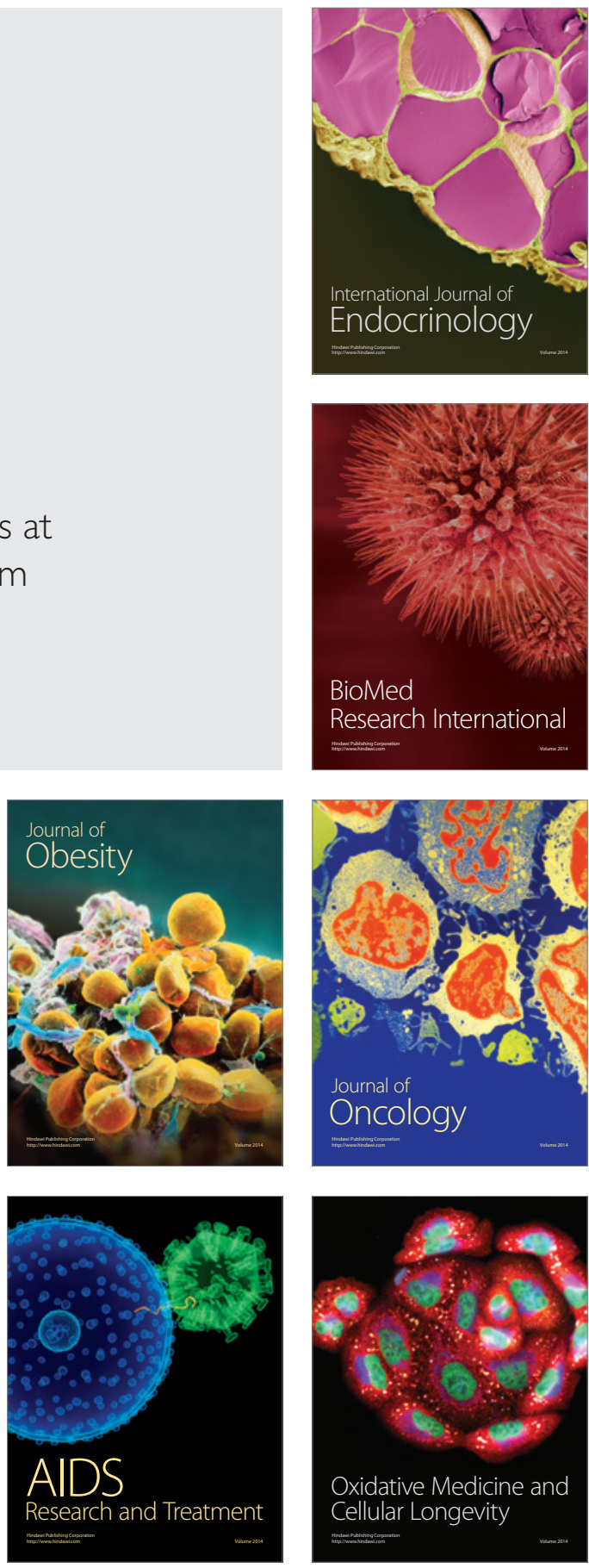